\title{
Use of Continuous Sedation Protocol Versus Interrupted Sedation Protocol in COPD Patients Mechanically Ventilated with Acute Respiratory Failure
}

\author{
Y.E.Rezk , T.S.Essawy, B.M.Aglan and W.A.El-kholy \\ Cardiothoracic Surgery \& Chest Diseases Dept., Faculty of Medicine, Benha Univ., Benha, Egypt
}

E-Mail: Elkholy@gmail.com

\begin{abstract}
Critically ill patients who require mechanical ventilation are often given contiouous intravenous infusions of sedative drugs to treat anxiety. Tranquilizers are mixed ceaselessly as contrasted and discontinuous bolus imbuement, this methodology gives a more steady degree of sedation and may increment patients'comfort, yet it expanded sedation may restrict clinicians capacity to decipher physical assessments. Be that as it may, every day interference of narcotic implantations to permit patients to "wake up" may improve the circumstance by permitting clinicians to smooth out the organization of tranquilizers while guaranteeing ideal solace for patients. To think about between consistent sedation convention versus intruded on sedation convention in COPD precisely ventilated patients as for span of mechanical ventilation, the time required for patients to stir, and the recurrence of unfriendly occasions. This examination was completed on 45 subjects with signs and manifestations of COPD and demonstrated radiologically; classified into three gatherings; 15 precisely ventilated COPD patients got persistent sedation convention [group 1], 15 precisely ventilated COPD patients with day by day interference sedation convention [group 2] and 15 precisely ventilated COPD patients without convention of sedation [group 3]. Term of mechanical ventilation was altogether shorter in intruded on sedation gathering and gathering without convention of sedation. Likewise, the ideal opportunity for patients to stir was essentially shorter in intruded on sedation gathering and gathering without convention of sedation. Every day interference sedation convention limits the confusions from total sedation, without an expansion in unfavorable occasions or different inconveniences.
\end{abstract}

Keywords: Chronic obstructive pulmonary disease, Respiratory failure, Mechanical ventilation, Sedation.

\section{Introduction}

Chronic Obstructive Pulmonary Disease [COPD], a typical preventable and treatable ailment, is portrayed by steady wind current restriction that is normally dynamic and related with an upgraded incessant incendiary reaction in the aviation routes and the lung to poisonous particles or gases. Intensifications and comorbidities add to the general seriousness in individual patients [1].

Respiratory disappointment is a typical and significant occasion, which is as often as possible related with extreme intensifications of interminable obstructive pneumonic malady [COPD]. The issue of respiratory disappointment in the COPD tolerant who turns out to be intensely sick may speak to a disintegration in the patient's premorbid condition with the end goal that hypoxaemia intensifies and hypercapnia creates during a generally trifling respiratory lot contamination, which might be viral or bacterial [2].

It is fitting to streamline these patients preoperatively to stay away from intricacies in the postoperative period the same number of times mechanical ventilation can be to a greater extent an issue than an answer. Serious COPD intensifications requiring mechanical ventilation speak to an extensive level of ICU affirmations. Ventilating a COPD tolerant is regularly troublesome on the grounds that the sickness might not have a reversible part. Sedation is important to encourage intubation, spend smooth remain on the ventilator, and maintain a strategic distance from asynchrony [3].
Azis H [4] analyzed constant sedation and DSI in precisely ventilated kids with deference toduration of mechanical ventilation, the time required for patients to stir, and the recurrence of unfavorable occasions. They reasoned that the term of mechanical ventilation and the ideal opportunity for patients to stir are fundamentally diminished in the day by day sedation interference bunch contrasted with the constant sedation gathering.

The point of the investigation is to analyze between constant sedation convention versus every day interference sedation convention in COPD precisely ventilated patients and the out happen to patient and term of mechanical ventilation and stay in ICU.

\section{Patients and methods}

This prospective study was carried out at Critical Care Department, Benha University Hospital and Benha Teaching Hospital on 45 COPD patients of both genders in age range of 18 to 80 years if they required mechanical ventilation for an expected duration of at least 24 hours and needed sedative drugs.

They were admitted in intensive care unit. They were divided equally into 3 groups according to:

- Group 1: 15 mechanically ventilated COPD patients received continuous sedation protocol.

- Group 2: 15 mechanically ventilated COPD patients with daily interruption sedation protocol.

- Group 3: 15 mechanically ventilated COPD patients without protocol of sedation. 


\section{Dosing adjustment}

- Midazolam: Initial intravenous bolus of $0.5-5$ mg every 1-5 minutes as needed.

- Midazolam: Continuous infusion at $1-2 \mathrm{mg} / \mathrm{hr}$; dosage to be increased in increments of $1-2$ $\mathrm{mg} / \mathrm{hr}$ until adequate sedation is achieved.

- Propofol: Continuous infusion at $5 \mu \mathrm{g} / \mathrm{kg}$ of body weight $/ \mathrm{min}$; dosage to be increased in increments of $5-10 \mu \mathrm{g} / \mathrm{kg} /$ minute every $2 \mathrm{~min}$ until adequate sedation is achieved.

In group 1, continous protocol continues to 6 hours before planned weaning from mechanical ventilation. In group 2, daily interruption of sedation for few hours until assessment of consciousness level. In group 3, giving patient bolus dose when become agitated on ventilator.

\subsection{Inclusion criteria}

Patient were known to be COPD diagnosed before with acute respiratory faliure with history of risk factors and required mechanical ventilation for an expected duration of at least 24 hours.

\subsection{Exclusion criteria}

- Age less than 18 years.

- Allergy or intolerance to used drug in each group.

- Liver cirrhosis.

- Renal failure.

- COPD patient on NIPPV.

- Shock stage.

- Coma of neurological origin.

\subsection{Methods}

\subsubsection{History taking}

- History of smoking.

- History of chest symptoms.

- History of any other co-morbidities as ischemic heart diseases, hypertension, diabetes mellitus, tuberculosis, malignancy, hepatic cirrhosis, endstage renal disease, rheumatoid arthritis and any systemic infection or inflammation.

\subsubsection{Clinical examination}

Complete physical examination was done with emphasis on vital signs.

\subsubsection{Laboratory investigations}

1. Complete Blood Count $[\mathrm{CBC}]$ during stable and exacerbation stage to assess platelets, neutrophils and lymphocytes [total and differential].

2. Urea.

3. $\mathrm{ABG}$

4. SGPT.

\subsubsection{Radiological investigations}

Chest X-ray and CT chest.

\subsubsection{Outcome}

The duration of mechanical ventilation was the primary outcome, while the time for patients to awaken on sedative infusion and the frequency of adverse events were secondary outcomes.

\subsection{Statistical analysis}

Data analysis was done using SPSS for Windows statistical package, version 24. Data were analyzed by intention to treat. Descriptive data were presented as percentages, mean [SD], and median [interquartile range]. The three study groups were compared using independent T-test or Mann Whitney U test. All tests were two-tailed and $\mathrm{P}$ values $<0.05$ were statistically significant.

\section{Results}

Patients age in the continuous sedation group was greater than other Groups, where the median was 58, 55 , and 50 respectively, and the mean was 53,49 , and 47 respectively Table (1).

Male Percentage in all groups was greater than Female percentage, but the highest percentage was in the continuous sedation group $67 \%$ male compared with $33 \%$ female Fig (1).

Regarding smoking statue, the percentage of current smokers was greater in all groups, patients without protocol of sedation included the highest percentage of current smokers $60 \%$, and interrupted sedation group was the greater percentage of current nonsmokers $40 \%$ Fig (2).

All groups consisted of higher percentage from good nutrition 60,53, and 67 respectively than malnourished Fig (3).

For three groups, $\mathrm{CO} 2$ narcosis is the major Indication for $\mathrm{MV}$, then severe respiratory distress, cardiorespiratory arrest after that, and finally the other indication was the lowest percentage as Indication for MV Fig (4).

Median [range] duration of mechanical ventilation was significantly shorter in interrupted sedation group compared to the continuous sedation group Mean [ \pm $\mathrm{SD}$ ] duration of mechanical ventilation was significantly shorter in interrupted sedation group compared to the continuous sedation group. The time for patients to awaken was also significantly lower in interrupted sedation group than in the continuous sedation group. The frequencies of adverse events were similar in both groups, because $\mathrm{P}$-value greater Than $0.05[\mathrm{p}=0.456]$ Table (2).

Median [range] duration of mechanical ventilation was significantly shorter in group without protocol of sedation compared to the continuous sedation group. Mean [ $\pm \mathrm{SD}$ ] duration of mechanical ventilation was significantly shorter in group without protocol of sedation compared to the continuous sedation group. The time for patients to awaken was also significantly lower in in group without protocol of sedation than in the continuous sedation group. The frequencies of 
adverse events were similar in both groups, because Pvalue greater than $0.05[\mathrm{p}=0.099]$ Table (3).

Median [range] and Mean [ \pm SD] duration of mechanical ventilation was similar in Interrupted sedation protocol group compared to group without protocol of sedation [p $=0.868]$. The time for patients to awaken was also similar in Interrupted sedation protocol group compared to group without protocol of sedation [ $p=0.395]$. The frequencies of adverse events were similar in both groups [ $\mathrm{p}=0.197]$ Table (4).

Table (1) Baseline characteristics of study subjects.

\begin{tabular}{lcccc}
\hline & Characteristics & Group 1 & Group 2 & Group 3 \\
& & {$[\mathbf{N}=15]$} & {$[\mathbf{N = 1 5}]$} & {$[\mathbf{N = 1 5}]$} \\
\hline \multirow{3}{*}{ Age } & Median age, [range] & $58[18-$ & $55[18-$ & $50[18-$ \\
& Mean age [years] \pm SD & $53 \pm 24$ & $49 \pm 19$ & $47 \pm 19$ \\
\hline
\end{tabular}

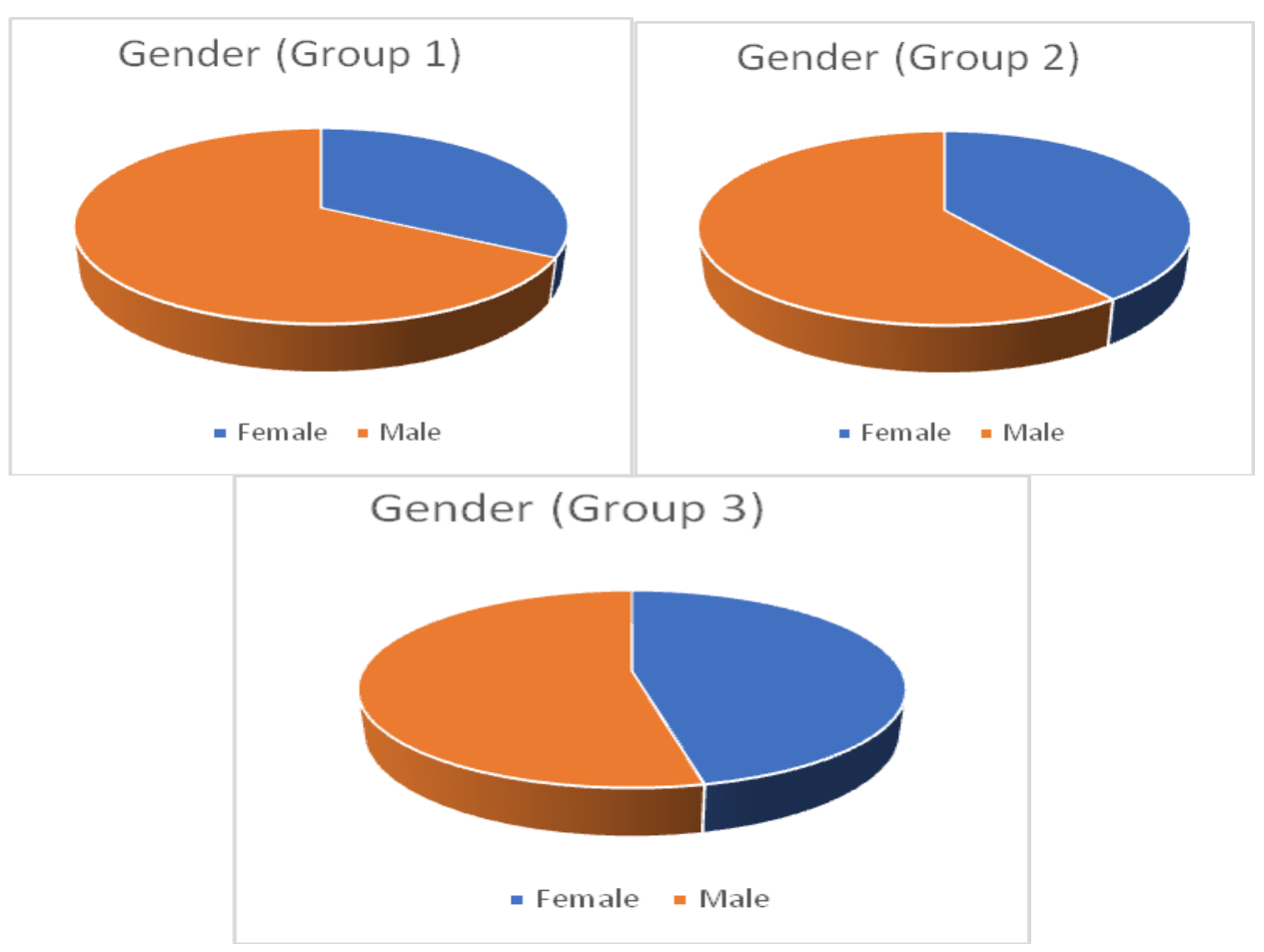

Fig (1) Percentage of male and female in each group.

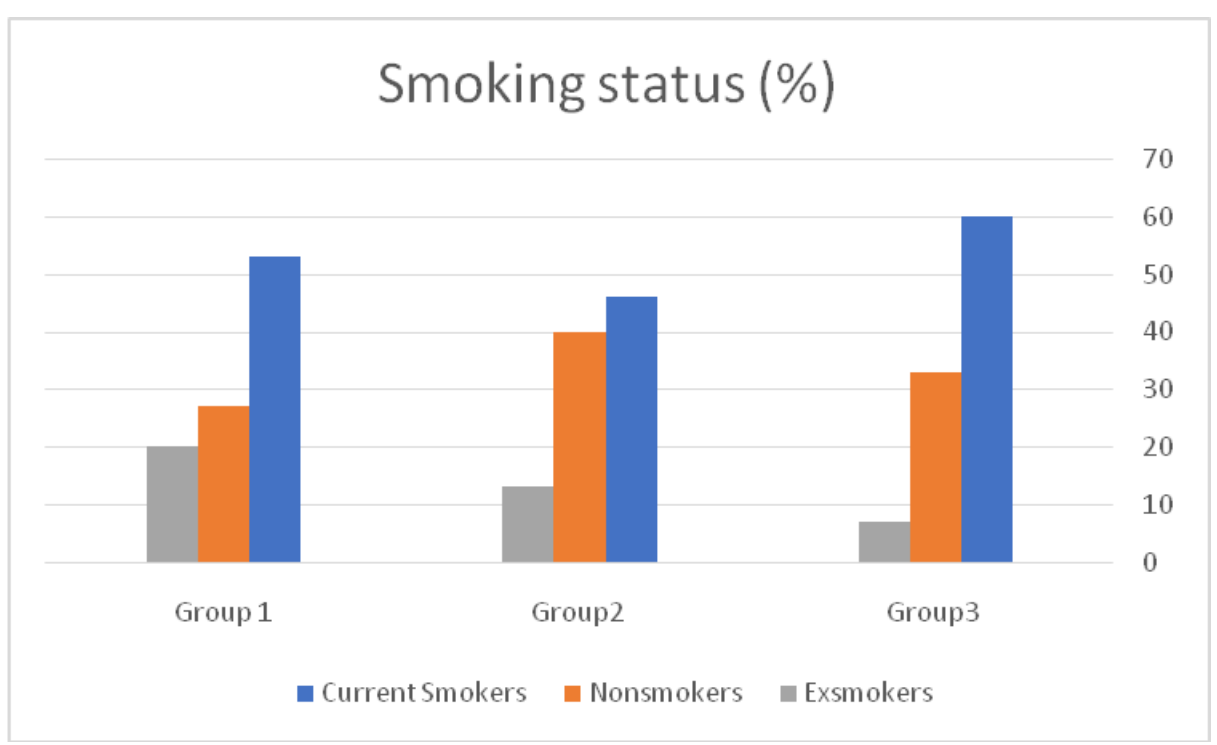

Fig (2) Percentage of smoking status 


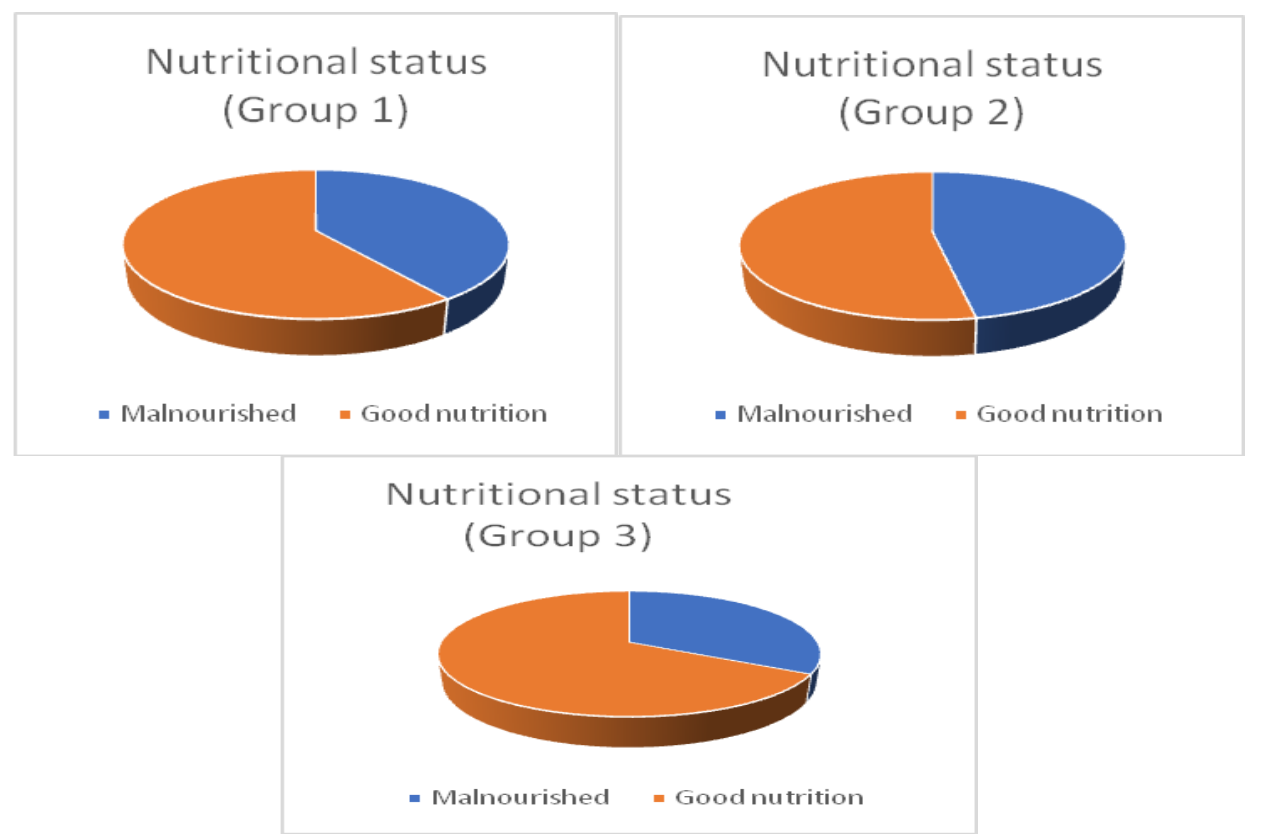

Fig (3) Percentage of nutritional status.

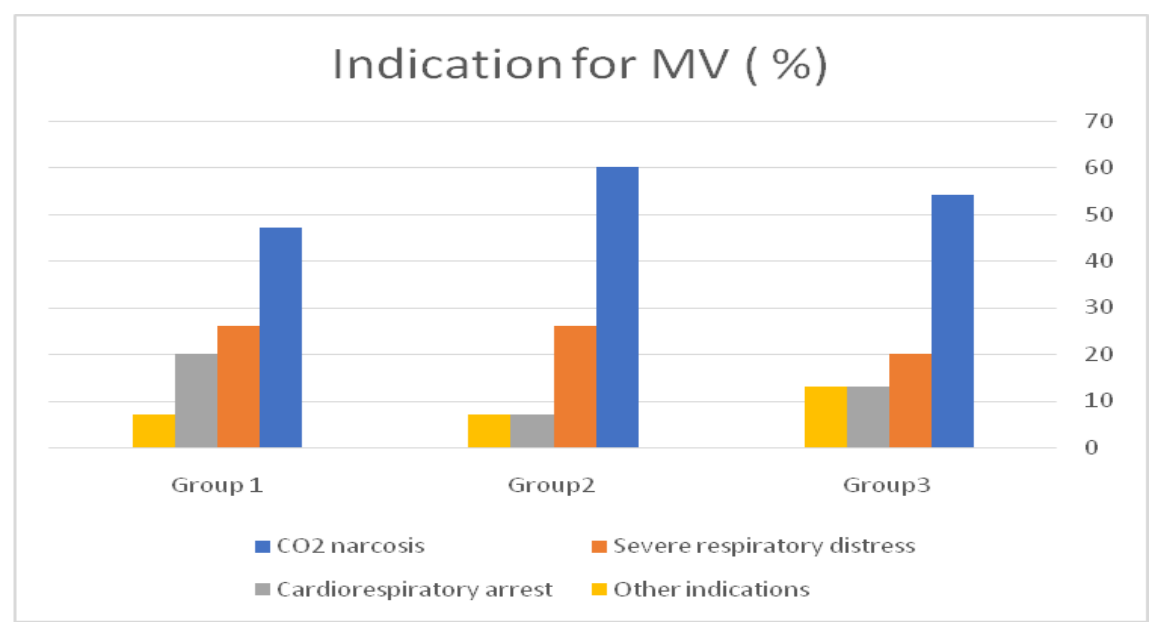

Fig (4) Indication for MV [\%].

Table (2) Continuous sedation group versus interrupted sedation protocol group: primary and secondary outcomes.

\begin{tabular}{lcccc}
\hline Variable & Group [1] & $\begin{array}{c}\text { Group [2] } \\
\text { [n=15] }\end{array}$ & $\begin{array}{c}\mathbf{p} \\
\text { value }\end{array}$ \\
\hline length of mechanical & Mean + SD & $79 \pm 34$ & $48 \pm 12$ & 0.013 \\
ventilation, hours & Median [range] & $65[63-130]$ & $44[36-75]$ & \\
Time for patients to & Mean \pm SD & $64 \pm 28$ & $44 \pm 17$ & 0.034 \\
awaken, hours & Median [range] & $57[30-120]$ & $44[24-77]$ & \\
& Spontaneous extubation & $4[27]$ & $3[20]$ & 0.456 \\
Adverse events & Hypotension & $1[7]$ & $2[13]$ & $2[13]$ \\
[n \%] & Accidental removal of & $3[20]$ & & \\
\hline
\end{tabular}

Table (3) Continuous sedation group versus group without protocol of sedation: primary and secondary outcomes.

\begin{tabular}{lcccc}
\hline Variable & & Group [1] & Group [3] & p value \\
\hline $\begin{array}{l}\text { length of mechanical } \\
\text { ventilation, hours }\end{array}$ & Mean \pm SD & $79 \pm 34$ & $46 \pm 8$ & 0.006
\end{tabular}


Table (3) Continue

\begin{tabular}{|c|c|c|c|c|}
\hline $\begin{array}{l}\text { Time for patients to } \\
\text { awaken, hours }\end{array}$ & $\begin{array}{c}\text { Mean } \pm \text { SD } \\
\text { Median [range] }\end{array}$ & $\begin{array}{c}64 \pm 28 \\
57[30-120]\end{array}$ & $\begin{array}{c}41 \pm 18 \\
33[24-70]\end{array}$ & 0.014 \\
\hline & Spontaneous extubation & $3[20]$ & $2[13]$ & \\
\hline Adverse events & Hypotension & $1[7]$ & $2[13]$ & 0.099 \\
\hline [n \%] & $\begin{array}{c}\text { Accidental removal of medical } \\
\text { equipment }\end{array}$ & $2[13]$ & $1[7]$ & 0.099 \\
\hline
\end{tabular}

Table (4) Interrupted sedation protocol group versus group without protocol of sedation: primary and secondary outcomes.

\begin{tabular}{lcccc}
\hline Variable & Group [2] & Group [3] & $\begin{array}{c}\text { p } \\
\text { value }\end{array}$ \\
\hline length of mechanical & Mean \pm SD & $48 \pm 12$ & $46 \pm 8$ & 0.868 \\
ventilation, hours & Median [range] & $44[36-75]$ & $41[36-61]$ & \\
Time for patients to & Mean \pm SD & $44 \pm 17$ & $41 \pm 18$ & 0.395 \\
awaken, hours & Median [range] & $44[24-77]$ & $33[24-70]$ & \\
& Spontaneous extubation & $3[20]$ & $2[13]$ & \\
Adverse events & Hypotension & $2[13]$ & $2[13]$ & 0.197 \\
[n \%] & Accidental removal of medical & $2[13]$ & $1[7]$ & \\
& equipment & & & \\
\hline
\end{tabular}

\section{Discussion}

Chronic obstructive Pulmonary Disease [COPD], a typical preventable and treatable ailment, is portrayed by diligent wind stream impediment that is generally dynamic and related with an upgraded interminable incendiary reaction in the aviation routes and the lung to toxic particles or gases. Intensifications and comorbidities add to the general seriousness in singular patients. The fundamental driver of horribleness and mortality among COPD patients are cardiovascular ailment [CVD] cellular breakdown in the lungs, and osteoporosis [1].

COPD is related with expanded fundamental and aviation route irritation, and improved aggravation declines clinical manifestations and diminishes lung capacity of patients, requiring hospitalized treatment [5].

Ventilated Patients are frequently calmed so as to forestall distress or nervousness and to encourage treatment. Portions are independently titrated, in view of sedation evaluations, to arrive at the ideal degree of sedation. Both deficient and unnecessary sedation may have malicious impacts. Over-sedation postpones recuperation, advances resistance, expands the term of mechanical ventilation, and prompts troubling side endless supply of the medications. Under-sedation may bring about expanded misery and expanded antagonistic occasions, for example, impromptu extubation, unintentional dislodging of catheters, and battling the ventilator [6].

Day by day sedation interference [DSI] convention is a compelling and safe technique to diminish the term of mechanical ventilation [7]. The DSI improves clinical results and results in a critical diminishing in the span of mechanical ventilation [8].

Notwithstanding, restricted information have been introduced on the sedation convention in COPD precisely ventilated patients. The point of this work was to look at between ceaseless sedation convention versus intruded on sedation convention in COPD precisely ventilated patients concerning span of mechanical ventilation, the time required for patients to stir, and the recurrence of unfavorable occasions.

This investigation was done on 45 subjects of both genders, who were introduced to Critical Care Department, Benha University Hospital and Benha Teaching Hospital. Patients were given signs and side effects of COPD and demonstrated radiologically, as per the GOLD rules, in view of supported [ 48 hours or more] intensifying of dyspnea, hack, or sputum creation prompting an expansion in the utilization of upkeep prescriptions or supplementations with extra drugs.

Subjects were arranged into three gatherings; 15 precisely ventilated COPD patients got constant sedation convention [group 1], 15 precisely ventilated COPD patients with day by day interference sedation convention [group 2] and 15 precisely ventilated COPD patients without convention of sedation [group 3].

Patients in which we foreseen demise to happen inside 24 hours or who were booked for withdrawal of life uphold, patients whose degree of sedation couldn't be scored because of basic neurologic condition, patients who had encountered cardiopulmonary revival, patients who utilized ceaseless tranquilizers for seizure treatment, and patients who were admitted to the division of basic consideration after already having utilized mechanical ventilation or narcotic medications were barred.

In this investigation, all the gatherings were coordinated regarding age, sex, smoking status, healthful status, and signs for MV. Tolerant age in the constant sedation bunch was more prominent than different gatherings, where the middle was 58,55 , and 
50 individually, and the mean was 53, 49, and 47 separately. Male Percentage in all gatherings was more prominent than female rate, yet the most noteworthy rate was in the nonstop sedation bunch $[67 \%$ male contrasted and $33 \%$ female].

H.Azis [4] contrasted DSI with ceaseless organization of narcotic imbuement in precisely ventilated kids. Forty patients were randomized into two gatherings, 18 kids in the constant sedation bunch [group A] and 22 in the DSI bunch [group B]. Both the gatherings were coordinated as for age and sex. [9] found that there were 200 men and 103 ladies, and the mean age was 61 years.

Concerning status, the level of current smokers was more prominent in all gatherings, patients without convention of sedation incorporated the hihgest level of current smokers [60\%], and intruded on sedation bunch was the more noteworthy level of current nonsmokers [40\%].

All gatherings comprised of higher rate from great sustenance $[60 \%, 53 \%$, and $67 \%$ respectively] than malnourished patients. For the three gatherings, $\mathrm{CO} 2$ narcosis is the significant sign for $\mathrm{MV}$, at that point extreme respiratory pain, cardiorespiratory capture. Different signs were the most minimal rate as signs for MV. Azis et al. H.Azis [4] found that both the gatherings were coordinated as for beginning ventilation factors.

In our investigation, span of mechanical ventilation was fundamentally shorter in intruded on sedation gathering and gathering without convention of sedation contrasted with the ceaseless sedation gathering. Notwithstanding, span of mechanical ventilation was comparative in intruded on sedation convention bunch contrasted with bunch without convention of sedation.

Our discoveries were in understanding with Kress JP [10] who demonstrated noteworthy decreases in the lengths of ventilation and ICU remain in grown-up patients. They exhibited an abatement in the middle ICU length of remain to 3.5 days in the interfered with sedation gathering, while.[11] found that convention based sedation was better as it indicated a decrease in the length of ICU remain from 19.9 to 14.0 days.

K.Gupta [12] indicated additionally that DSI improved results in pediatric patients. The length of mechanical ventilation was essentially decreased in the intruded on sedation bunch contrasted with the constant sedation group.. H.Azis [4] found that the span of mechanical ventilation was fundamentally longer in the nonstop sedation bunch contrasted with the DSI gathering. Notwithstanding, it ought to be noticed that these examinations were in kids.

In our examination, the ideal opportunity for patients to stir was fundamentally shorter in intruded on sedation gathering and gathering without convention of sedation contrasted with the nonstop sedation gathering. Be that as it may, the ideal opportunity for patients to stir was comparative in intruded on sedation convention bunch contrasted with bunch without convention of sedation.
K.Gupta [12] announced that the level of days a patient was alert in the constant gathering was altogether lower contrasted with that in the intruded on sedation gathering. Also,.[4] found that the ideal opportunity for patients to arousing the nonstop gathering was essentially longer contrasted with the DSI gathering.

In our examination, the frequencies of antagonistic occasions were comparative in the gatherings with inconsequential contrast. Also, H.Azis [4] found that the quantity of patients who created antagonistic occasions were comparative in the two gatherings. Three patients created hypotension, one in bunch An and two in bunch B. A sum of four scenes of unconstrained extubation were recorded, two in each gathering. Two scenes of coincidental expulsion of clinical hardware were noted, one in each gathering. In any case, it ought to be noticed that these investigations were in kids.

S.Mehta [13] contrasted protocolized sedation and protocolized sedation in addition to day by day sedation break in basically sick patients. For fundamentally sick patients accepting mechanical ventilation, when attendants actualized a sedation convention that focused light sedation, day by day sedation interference didn't lessen the term of mechanical ventilation, offered no extra advantages for patients, and may have expanded both sedation and pain relieving use and medical caretaker outstanding burden.

AF Alkarn [14] tried the adequacy and wellbeing of no-sedation convention in controlling COPD patients' boundaries on a ventilator. They inferred that no-sedation procedure is related with fundamentally higher $\mathrm{PaCO} 2, \mathrm{HR}, \mathrm{MAP}$, and respiratory rate in COPD precisely ventilated patients. No noteworthy distinction was found in respiratory muscle work. Limiting sedation may not influence the general patient result yet may effectsly affect understanding solace on the ventilator.

Henceforth, conventions pointed toward limiting the complexities of total sedation have been created. In such manner, every day interference of narcotic mixtures was discovered to be a superior alternative, as it decreased the term of mechanical ventilation. The length of mechanical ventilation is a significant determinant of results in ventilated patients [15].

\section{Conclusion}

The length of mechanical ventilation and the ideal opportunity for patients to arousing the constant gathering are essentially longer contrasted with the other two gatherings [interrupted sedation convention gathering and gathering without convention of sedation].

The span of mechanical ventilation and the ideal opportunity for patients to stir are comparable in bunch without convention of sedation and intruded on sedation convention gathering. 
The number of patients who created unfriendly occasions are comparable in all gatherings.

The day by day interference sedation convention limits the confusions from combined sedation, without an expansion in antagonistic occasions or different difficulties.

\section{References}

[1] Global Initiative For Chronic Obstructive Lung Disease [GOLD]. Global strategy for the diagnosis, management, and prevention of COPD Updated 2017. Belgium: Global Initiative For Chronic Obstructive Lung Disease, Inc, 2018.

[2] S.Sethi, N.Evans, Grant BJB, et al. New strains of bacteria and exacerbations of chronic obstructive pulmonary disease. N Engl J Med, , Vol.347, PP.465-471,2002.

[3] KM.Liao, YC.Chen, KC.Cheng, et al. Trends in intensive care unit admissions of COPD patients from 2003 to 2013 in Taiwan. Int J Chron Obstruct Pulmon Dis, Vol.13, 2007.

[4] H.Azis, S.Triratna and E.Bahar, Continuous sedation versus daily sedation interruption in mechanically-ventilated children. Paediatr Indones, Vol.56 (1), PP. 19-23,2016.

[5] X.Zhou, Q.Li and X.Zhou, Exacerbation of chronic obstructive pulmonary disease. Cell Biochem Biophys., Vol.73(2), PP. 349-355,2015.

[6] NJ.Vet, de Wildt SN, Verlaat CWM, et al. Daily interruption of sedation in critically ill children: study protocol for a randomized controlled trial. Trials, Vol.15, PP.55,2014.

[7] CW.Verlaat, GP.Heesen, NJ.Vet, et al. Randomized controlled trial of daily interruption of sedatives in critically ill children. Pediatr Anesth., Vol.24, PP.151-6,2014.

[8] Y.Shehabi, R.Bellomo, S.Mehta, et al. Intensive care sedation: the past, present and the future. Crit Care., Vol.17, PP. 322-9,2013.

[9] Yao CY, Liu XL and Tang Z. Prognostic role of neutrophil-lymphocyte ratio and plateletlymphocyte ratio for hospital mortality in patients with AECOPD. International J.,COPD, Vol.12, PP.2285-2290,2017.

[10] JP.Kress, AS.Pohlman, MF.O'Conner, et al. Daily interruption of sedative infusions in critically ill patients undergoing mechanical ventilation. N Engl J Med, Vol.342, PP.1471-7,2000.

[11]AD.Brook, TS.Ahrens, R.Schaiff R, et al. Continuous vs. daily sedation interruption in children. Paediatr Indones., Vol.56(1), 2016.

[12]K.Gupta, VK.Gupta, M.Jayashree, et al. Randomized controlled trial of interrupted versus continuous sedative infusions in ventilated children. Pediatr Crit Care Med, Vol.13, PP.1315,2012 .

[13] S.Mehta, L.Burry, D.Cook, et al. Daily sedation interruption in mechanically ventilated critically ill patients cared for with a sedation protocol: A randomized controlled trial. JAMA, Vol.308(19), PP.1985-,1992.

[14] AF.Alkarn, MF.Abdel Ghany, MGA.Khalaf, et al. Effects of sedation on clinical, gasometric, and respiratory muscle parameters in critically ill chronic obstructive pulmonary disease patients. Egyptian J.,Bronchology, 2019.

[15] PN.Johnson, JL.Miller TM.Hagemann, Sedation and analgesia in critically ill children. AACN Adv Crit Care. Vol.23 , PP.415-34,2012. 